\title{
Gain and Bandwidth Enhancement of Microstrip Antenna Array using Double Square Split Ring Resonator FSS as a Superstrate for WiMax/WiFi/WLAN Applications
}

\author{
V. Rajya Lakshmi \\ Professor, Dept. of ECE \\ ANITS, Visakhapatnam \\ 531162, India
}

\author{
G. Viswanadh \\ Raviteja \\ Assistant Professor, Dept. \\ of ECE \\ ANITS, Visakhapatnam \\ 531162, India
}

\author{
G. Sujatha \\ Department of ECE \\ ANITS, Visakhapatnam \\ 531162, India
}

\author{
K. Balasagar \\ Department of ECE \\ ANITS, Visakhapatnam \\ 531162, India
}

\author{
K. V. Tejaswi \\ Department of ECE \\ ANITS, Visakhapatnam \\ 531162, India
}

\author{
A. L. S. Yashwanth \\ Department of ECE \\ ANITS, Visakhapatnam \\ 531162, India
}

\author{
K. Sai Teja \\ Department of ECE \\ ANITS, Visakhapatnam \\ 531162, India
}

\begin{abstract}
Microstrip patch antennas are very popular in wireless communication because of its low profile, lightweight, low production cost. However, the gain and bandwidth of the microstrip patch antenna are very less. The main objective of this paper is to enhance the gain of the microstrip antenna by using frequency selective surfaces as a superstrate. In this paper, a microstrip array antenna with 2 elements is proposed. To increase the bandwidth, a partial ground plane method is used. Also the comparison of two FSS layers with different shapes i.e. Square loop and Double Square Split Ring is performed. By using a partial ground plane, the bandwidth has been increased by $87 \%$. The gain of microstrip patch antenna array is calculated to be $4.43 \mathrm{~dB}$ by using square loop FSS whereas by using Double Square SRR, it is found to be 7.61 $\mathrm{dB}$. The proposed antenna outperformed the square loop FSS and with a bandwidth of $1.74 \mathrm{GHz}$ which can be used in number of wireless applications such as WiMax, WiFi, etc.
\end{abstract}

\section{General Terms}

Antenna Performance, Gain characteristics

\section{Keywords}

FSS, WLAN, Superstrate, WiMax, SRR, Microstrip antenna array.

\section{INTRODUCTION}

WLAN based wireless networks are specific to accessing the internet. A microstrip patch antenna which operates in the frequency range of 2 to $3 \mathrm{GHz}$ that can be used for WLAN applications is seen in [1]. There are different kinds of antennas that to choose but it should match with desired characteristics of a broadband antenna. Therefore, a microstrip patch antenna is chosen because it has many advantages and applications such as low cost, minimum weight and simple geometry [2]. A microstrip patch antenna is a metallic strip or patch mounted on a dielectric layer over a ground plane. The patch is designed to be of any shape. This antenna is used in satellite communication systems, GPS Systems, wireless LAN's, etc.[3,4]. In contrast, microstrip patch antenna has a narrow bandwidth, low gain, low directivity, and low radiation efficiency. To overcome the drawbacks of low gain for a conventional microstrip patch antenna, arrays can be used. By using antenna array, the parameters like gain, bandwidth, radiation efficiency, etc can be improved [5-7]. But the problem with antenna arrays is with occupied space and to increase gain and directivity further, the number of elements in an array should also be increased which results in an increase of structural dimensions.

Therefore, the preferable solution is the application of Frequency Selective Surfaces (FSS). These are periodic arrays of metallic patch or apertures on a dielectric slab and are used at specific or band of frequencies for reflecting fully (patch) or for transmitting (apertures) electromagnetic waves from the antenna [8-10]. In this paper, square loop FSS and Double SRR bandpass FSS as a superstrate are discussed which are mentioned in [11]. By applying frequency selective surfaces to microstrip antenna array of two elements, the radiation parameters which include bandwidth, gain and directivity have been improved significantly, when compared to conventional antenna array [12-16]. Hence the microstrip antenna array integrated with frequency selective surfaces is more efficient to obtain desired radiation characteristics.

\section{ANTENNA DESIGN}

\subsection{Conventional Microstrip Patch} Antenna

A simple microstrip inset fed antenna is designed which operates at $2-3 \mathrm{GHz}$ as shown in Fig 1 . The inset fed is used to improve the impedance matching between the element and the feed line. The dimensions of the patch are $\mathrm{L}=37.26 \mathrm{~mm}, \mathrm{~W}=$ $28.83 \mathrm{~mm}$. The substrate used was FR4 Epoxy with $\varepsilon \mathrm{r}=4.4$ and height of substrate is $1.6 \mathrm{~mm}$. The formulas for the design of Patch antenna by [1]. 


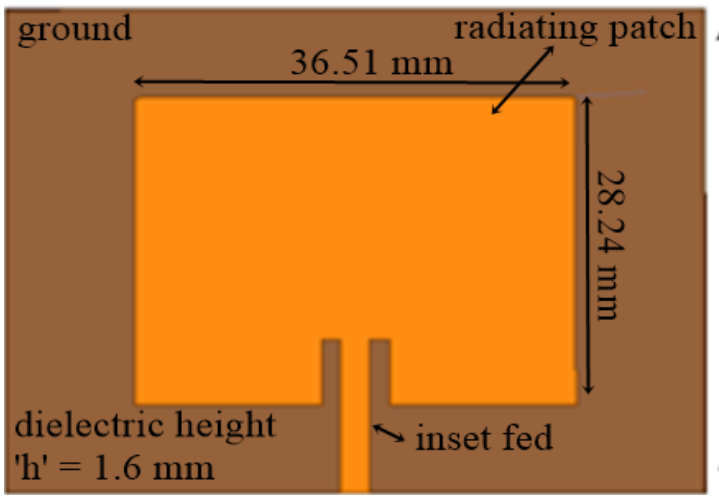

Fig. 1 Conventional Inset fed microstrip patch antenna design

\section{Equations of Patch Antenna:}

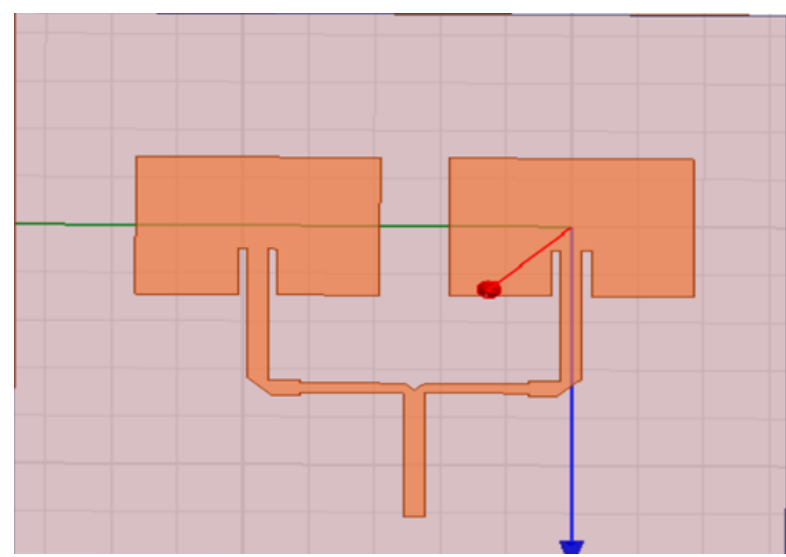

Fig. 3 Layout of microstrip patch antenna array

1. Width of the Patch, $\mathrm{W}=\frac{c}{2 f \sqrt{\frac{\varepsilon_{r+1}}{2}}}$

2. Effective Dielectric Constant, $\varepsilon_{\text {reff }}=\frac{\varepsilon_{r}+1}{2}+\frac{\varepsilon_{r}-1}{2}(1+$ $12 h w)-0.5$

(2)

Therefore, the actual increase in length $(\Delta L)$ of the patch is to be calculated as

$$
\frac{\Delta L}{h}=0.412 \frac{\left(\varepsilon_{r e f f}+0.3\right)\left(\frac{W}{h}+0.264\right)}{\left(\varepsilon_{r e f f}-0.258\right)\left(\frac{W}{h}+0.8\right)}
$$

Where, $\mathrm{h}=$ height of the substrate

3. Effective Length, $\mathrm{L}_{\mathrm{eff}}=\frac{c}{2 f \sqrt{\varepsilon_{\text {reff }}}}$

4. Length of the Patch, $\mathrm{L}=\mathrm{L}_{\mathrm{eff}}-2(\Delta L)$

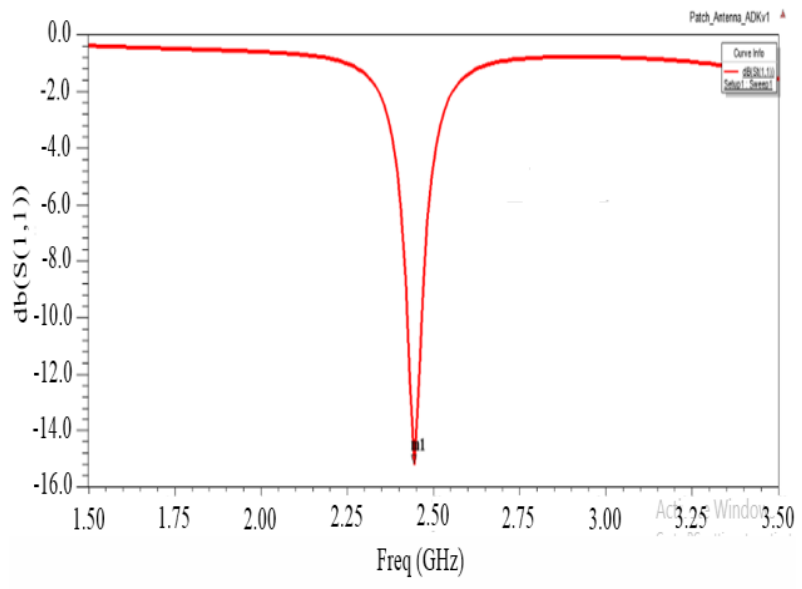

Fig. 2 Return loss plot for conventional microstrip patch antenna

From Fig. 2, S11 obtained is $-15.12 \mathrm{~dB}$. To improve the radiation parameters of the antenna, the antenna array is designed. The gain in this case was found to be $2.4 \mathrm{~dB}$.

\subsection{2 Design of Microstrip Array Antenna}

In this paper, an array with two elements is designed as shown in Figure 3. The designing of antennas with very high directive characteristics and to improve the gain and bandwidth, the most common configuration is the array form of microstrip antenna. Also for this work, corporate feed network is used in the designing of the microstrip antenna array.

The advantages of this corporate feed network include design simplicity, flexible choice of element spacing and broader bandwidth. It consists of transmission lines, bends, $\mathrm{T}$ junctions and impedance transformers. In this design, feed line is made with some discontinuities to compensate losses. Firstly, T-junction is used to divide the power for both the elements equally. The second discontinuity is Impedance Transformer which is used for impedance matching of different microstrip lines. Finally, the third discontinuity is bend which is used to compensate the losses. This is depicted in Fig. 3

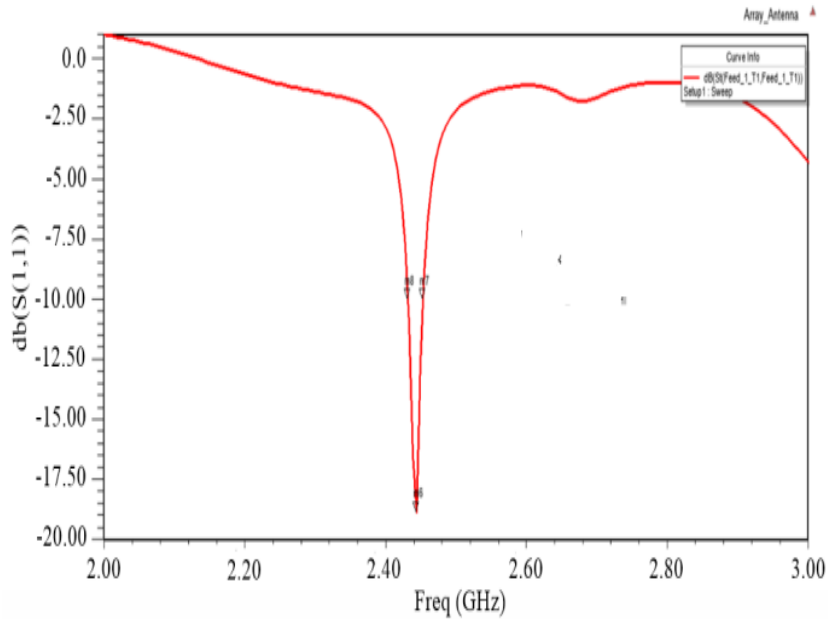

Fig. 4 Return loss plot for the microstrip antenna array 
Therefore, using the structural modifications and taking array characteristics into consideration, such as gain and directivity are enhanced to $2.40 \mathrm{~dB}$ and $3.67 \mathrm{~dB}$ respectively when compared to single Inset-fed patch antenna. From Fig. 4, the return loss obtained at the resonant frequency of $2.45 \mathrm{GHz}$ is $-18.16 \mathrm{~dB}$. But the bandwidth is considerably low.

\subsection{3 Partial ground plane for Proposed Array Antenna}

One method to enhance the bandwidth of microstrip antenna array is truncating the ground plane. This method of utilizing the concept of partial ground is discussed in [17]. The bandwidth obtained for the microstrip antenna array without truncating the ground plane is very much low. After using partial ground plane method, the bandwidth has been increased compared to the initial case of the array. But, by truncating the ground plane, the resonant frequency is reduced. To again increase the resonant frequency the dimensions of the antenna should be reduced. From Fig. 5, the dimensions of the antenna array with a truncated ground plane have been reduced, so that the resonant frequency is increased to the desired value.

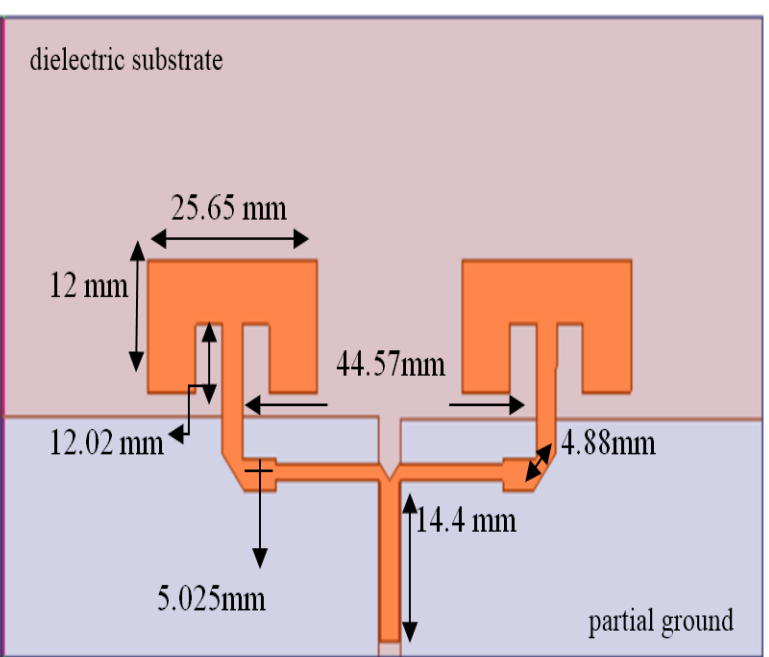

Fig. 5 Antenna Array with reduced dimensions

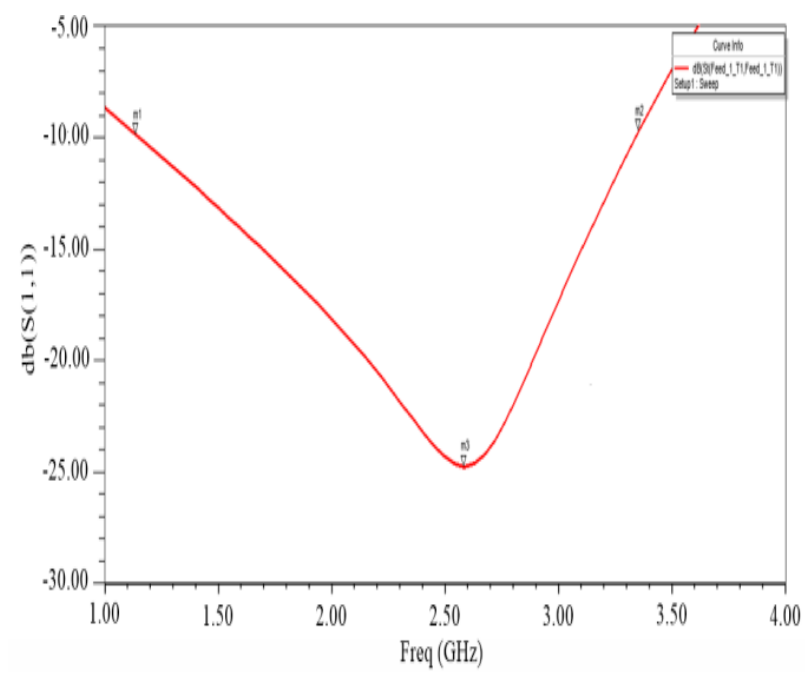

Fig. 6 Return loss plot for the antenna configuration with partial ground
From the above Fig. 6 , the resonant frequency increased to $2.58 \mathrm{GHz}$ and obtained return loss is $-24.7622 \mathrm{~dB}$ and the bandwidth achieved is $2181 \mathrm{MHz}$.

\section{FREQUENCY SELECTIVE SURFACES (FSS)}

Frequency Selective Surface is a thin repetitive surface designed to absorb or reflect the electromagnetic field based on frequency. The FSS has many applications such as used in Radomes, Stealth Technology, Dichroic sub-reflectors, etc. There are different types of FSS unit cell geometries. In this paper Square loop and Double square split ring resonator geometry are chosen. Therefore, in both cases Pass band FSS is designed. This layer is also known as Superstrate. FSS in general is not more than a LC circuit connected in series or parallel, to function just as a free space filter. So the superstrate layer is a series LC circuit.

FSS arrays are classified into two different type of elements: center connected, loop type. The first group, center connected type are used for radiating and non-radiating arrays. The loop elements are generally for non-radiating FSS arrays.

\subsection{Design of Unit Cell}

\section{A. Square loop}

An FSS layer is applied to conventional microstrip antenna array i.e. Bandpass FSS which acts as a superstrate. In this paper, the unit cell for bandpass is designed. While designing the unit cell, the Floquet port is used and the linked boundaries i.e. master and slave boundaries are used.

\section{Design consideration for Square loop (bandpass)}

The geometry of the proposed Square loop is as shown in Fig. 7. The substrate used is FR4 Epoxy with permittivity of 4.4 and loss tangent of 0.02. The thickness of the substrate is $1.6 \mathrm{~mm}$. Figure 7 (right) shows the 3D view of the unit cell. The dimensions of the Inner Square and outer square are $(16.85 \times 16.85) \mathrm{mm}$ and $(19 \times 19) \mathrm{mm}$.
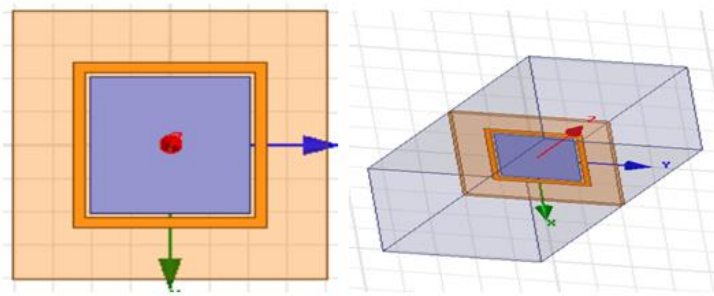

Fig. 7 (left) Top view of Unit Cell; (right) Side view of unit cell

The reflection coefficient of the unit cell is as shown in Figure 8. It shows that if an EM wave of $2.5 \mathrm{GHz}$ enters from port1 how much of it is reflected back. It can be seen that the designed cell transmits the frequencies in the range of 2.44 to $2.71 \mathrm{GHz}$ whereas the other frequencies are reflected. The unit cell resonates at $2.53 \mathrm{GHz}$ and $\mathrm{S} 11=-24.68 \mathrm{~dB}$. This is shown in Fig. 8. 


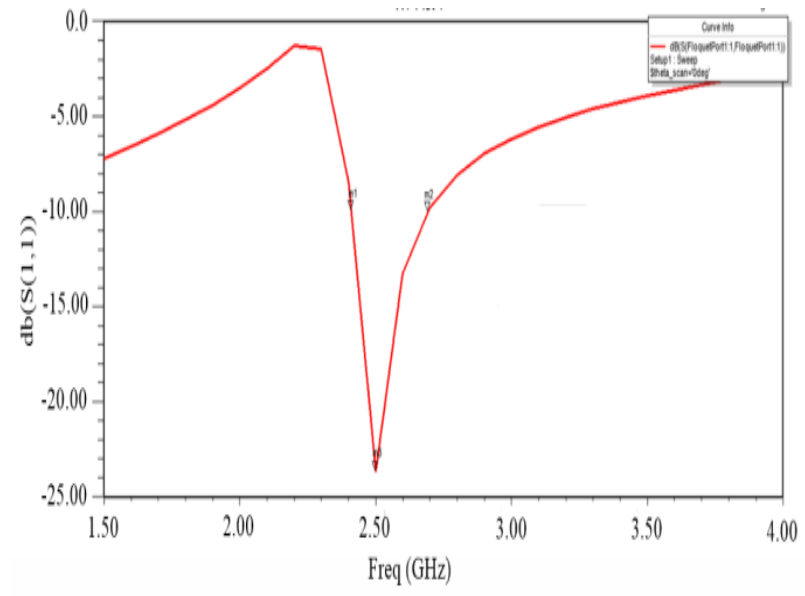

Fig. 8 Return loss plot for Square loop bandpass Unit Cell

\section{B. Double Square Split Ring Resonator}

The SRR may be modeled by an equivalent electrical circuit. This circuit behaves as a resonant cavity modeled by an LC circuit. The presence of a magnetic field $\mathrm{H}$ perpendicular to the plane separating the two split rings allows the creation of a gap capacitor $\mathrm{C}$ at the opening of each ring. The interaction between these two metal rings is through a mutual capacitor. In addition, each ring resembles a solenoid that can be represented by an inductance $\mathrm{Lm}$. The space between the two rings is modeled by a capacitor $\mathrm{Cm}$.

\section{Design of Double Square SRR}

The geometry of the proposed Double Square Split Ring Resonator is as shown in Figure 9. The substrate used is FR4 Epoxy with permittivity of 4.4 and loss tangent of 0.02 . The thickness of the substrate is $1.6 \mathrm{~mm}$. Fig. 9 (right) shows the $3 \mathrm{D}$ view of the unit cell.
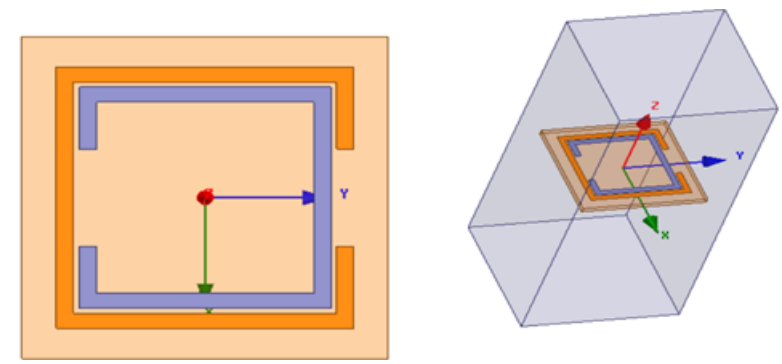

Fig. 9 (left) Double Square split ring resonator unit cell; (right) 3D view of Double Square SRR

The reflection coefficient of the unit cell is as shown in Fig. 10. It shows that if an EM wave of $2.5 \mathrm{GHz}$ enters from port1 how much of it is reflected back. It can be seen that the designed cell transmits the frequencies from 2.4 to $2.78 \mathrm{GHz}$.

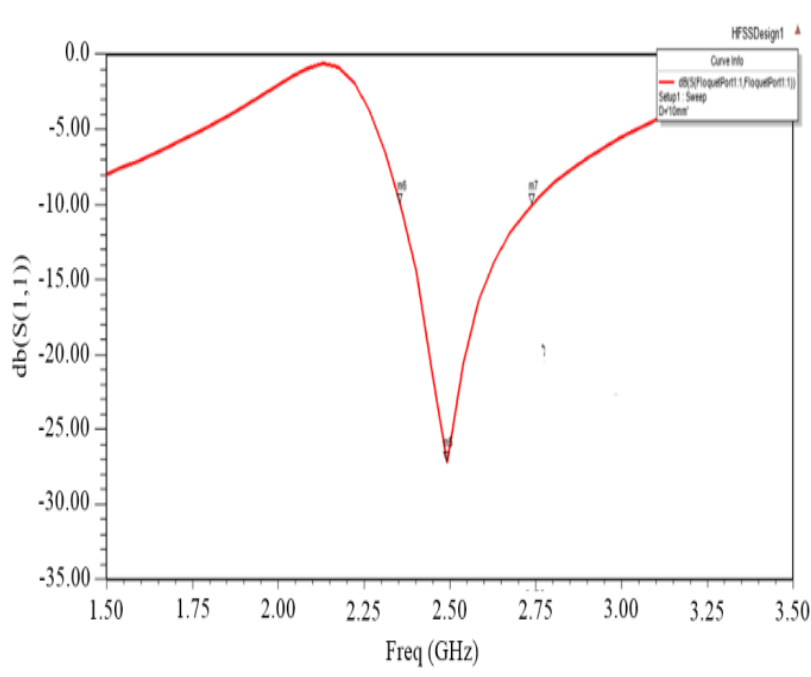

Fig. 10 Return loss plot for Double Square Split Ring Resonator bandpass Unit Cell

\subsection{FSS as a Superstrate}

\section{A. Square Loop}
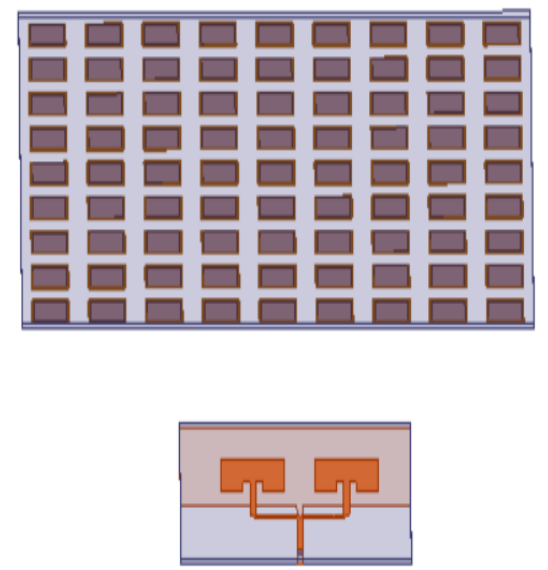

Fig. 11 Square loop bandpass FSS

The $9 \times 9$ array of square loop unit cell forming an FSS substrate is as shown in Fig. 11. This substrate is applied as a superstrate to the conventional microstrip antenna array. By the application of square loop bandpass as a superstrate, the gain and directivity will be significantly improved. The periodicity is of $20 \mathrm{~mm}$ in both $\mathrm{x}$ and $\mathrm{y}$ directions. 
By the application of Square Loop bandpass FSS as a superstrate the return loss $\mathrm{S} 11=-22.99 \mathrm{~dB}$, Gain is $4.43 \mathrm{~dB}$ and directivity is $7.62 \mathrm{~dB}$. This configuration even though proved to be satisfactory in terms of gain and directivity, the bandwidth was too less. This is shown in Fig. 12.

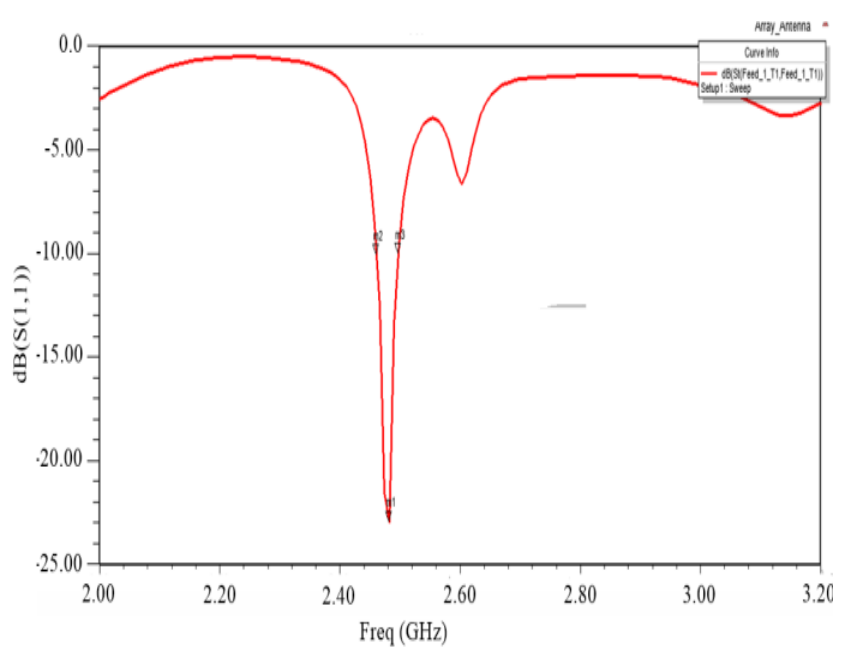

Fig. 12 Return loss plot of antenna array by using Square loop FSS as a superstrate.

\section{B. Double Square Split Ring Resonator}

After applying the two FSS layers to the array, the return loss, gain, and directivity have been improved but the bandwidth of the array was very less. To improve the bandwidth and further improvement of gain and directivity, another shape called Double Square Split Ring Resonator is chosen as shown in Fig. 13.

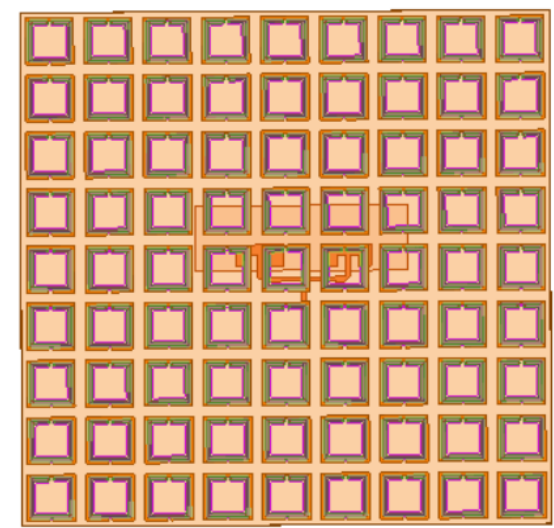

Fig. 13 Top view of the antenna array by using Double Square SRR as a superstrate.

The Double Square SRR has better results compared with the square loop FSS. The return loss is as shown below. By using Double Square SRR the bandwidth obtained is $1.7458 \mathrm{GHz}$ and gain and directivity is $7.61 \mathrm{~dB}$ and $8.52 \mathrm{~dB}$ respectively. The S11 return loss is $-37.43 \mathrm{~dB}$ with a center frequency of $2.86 \mathrm{GHz}$. These are plotted in Fig. 14.

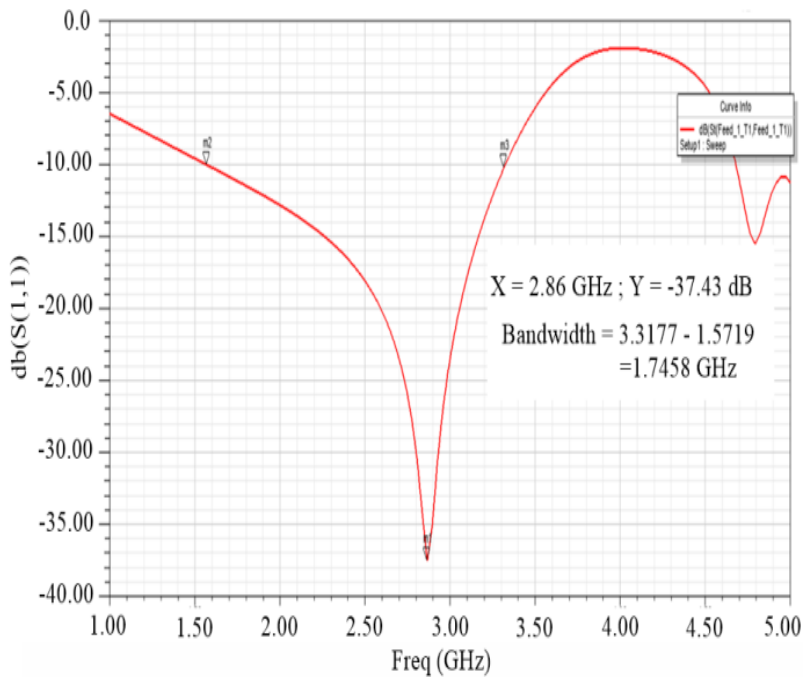

Fig. 14 Return loss plot of antenna array by using Double Square SRR as a superstrate.

Table 1 Comparison between antenna parameters for the two configurations

\begin{tabular}{|c|c|c|}
\hline Parameter & $\begin{array}{c}\text { Square loop FSS } \\
\text { as a superstrate }\end{array}$ & $\begin{array}{c}\text { Double Square } \\
\text { SRR FSS as } \\
\text { superstrate }\end{array}$ \\
\hline Gain & $4.43 \mathrm{~dB}$ & $7.61 \mathrm{~dB}$ \\
\hline Directivity & $7.62 \mathrm{~dB}$ & $8.52 \mathrm{~dB}$ \\
\hline Bandwidth & $0.14 \mathrm{GHz}$ & $1.74 \mathrm{GHz}$ \\
\hline S11 (dB) & $-22.99 \mathrm{~dB}$ & $-37.43 \mathrm{~dB}$ \\
\hline
\end{tabular}

\section{CONCLUSIONS}

This paper designs an FSS with two shapes i.e. Square loop and Double Square SRR which serves as a superstrate layer of the antenna and results are compared. By comparing the results, the application of Double Square SRR FSS to the antenna model is better than the Square loop FSS. The bandwidth of microstrip antenna is increased to $1.74 \mathrm{GHz}$ when compared to the narrow bandwidth constraints of the conventional microstrip patch antenna. Even though, square loop FSS is taken into consideration, the double square SRR FSS when used a superstrate outperformed the square loop FSS in every aspect. The results show that using the proposed FSS technique, the gain is enhanced to $7.61 \mathrm{~dB}$ and the bandwidth is increased to $1.74 \mathrm{GHz}$. Therefore, the designed antenna can be used for WLAN/WiFi/WiMax applications.

\section{REFERENCES}

[1] Almeida Filho, V.A. and Campos, A.L.P., 2014. Performance optimization of microstrip antenna array using frequency selective surfaces. Journal of Microwaves, Optoelectronics and Electromagnetic Applications, 13(1), pp.31-46.

[2] Balanis, C.A., 2016. Antenna theory: analysis and design. John wiley \& sons.

[3] Huque, M.T.I.U., Hosain, M.K., Islam, M.S. and Chowdhury, M.A.A., 2011. Design and performance analysis of microstrip array antennas with optimum 
parameters for X-band applications. International Journal of Advanced Computer Science and Applications, 2(4).

[4] Raviteja, G.V., 2018. Design and analysis of a novel dual trapezoidal slot-based rectangular microstrip antenna for wide area network using WiMax application. Microwave and Optical Technology Letters, 60(4), pp.1057-1060.

[5] Errifi, H., Baghdad, A., Badri, A. and Sahel, A., 2014, November. Design and simulation of microstrip patch array antenna with high directivity for $10 \mathrm{GHz}$ applications. In International Symposium on Signal Image Video and Communications, ISIVC-2014, Marrakech, Morocco (pp. 19-21).

[6] Anguera, J., Montesinos, G., Puente, C., Borja, C. and Soler, J., 2003. An undersampled high-directivity microstrip patch array with a reduced number of radiating elements inspired on the Sierpinski fractal. Microwave and optical technology letters, 37(2), pp.100103.

[7] Raviteja, G.V. and An, X., Band based 2 X 1 Microstrip Antenna Array with Combined $\mathrm{H}$ and Dual U Slot Design Operating at $11 \mathrm{GHz}$ Frequency. Communications, 7, pp.15-20.

[8] Munk, B.A., 2000. Frequency selective surfaces theory and design. john Wiley\&Sons. Inc.

[9] Lee, Y.J., Yeo, J., Mittra, R. and Park, W.S., 2005, July. Design of a frequency selective surface (FSS) type superstrate for dual-band directivity enhancement of microstrip patch antennas. In 2005 IEEE Antennas and Propagation Society International Symposium (Vol. 3, pp. 2-5). IEEE.

[10] Pirhadi, A., Bahrami, H. and Nasri, J., 2012. Wideband high directive aperture coupled microstrip antenna design by using a FSS superstrate layer. IEEE transactions on antennas and propagation, 60(4), pp.2101-2106.

[11] Ferreira, D., Caldeirinha, R.F., Cuiñas, I. and Fernandes, T.R., 2015. Square loop and slot frequency selective surfaces study for equivalent circuit model optimization. IEEE Transactions on Antennas and Propagation, 63(9), pp.3947-3955.

[12] Chen, H.Y., Tao, Y., Hung, K.L. and Chou, H.T., 2010, August. Bandwidth enhancement using dual-band frequency selective surface with Jerusalem cross elements for 2.4/5.8 GHz WLAN antennas. In 2010 IEEE International Conference on Wireless Information Technology and Systems (pp. 1-4). IEEE.

[13] Foroozesh, A. and Shafai, L., 2010. Investigation into the effects of the patch-type FSS superstrate on the high-gain cavity resonance antenna design. IEEE Transactions on Antennas and Propagation, 58(2), pp.258-270.

[14] Li, X.Y., Li, J., Tang, J.J., Wu, X.L. and Zhang, Z.X., 2015, December. High gain microstrip antenna design by using FSS superstrate layer. In 2015 4th International Conference on Computer Science and Network Technology (ICCSNT) (Vol. 1, pp. 1186-1189). IEEE.

[15] Lee, Y.J., Yeo, J., Mittra, R. and Park, W.S., 2005, July. Design of a frequency selective surface (FSS) type superstrate for dual-band directivity enhancement of microstrip patch antennas. In 2005 IEEE Antennas and Propagation Society International Symposium (Vol. 3, pp. 2-5). IEEE.

[16] Chen, H.Y., Tao, Y., Hung, K.L. and Chou, H.T., 2010, August. Bandwidth enhancement using dual-band frequency selective surface with Jerusalem cross elements for $2.4 / 5.8 \mathrm{GHz}$ WLAN antennas. In 2010 IEEE International Conference on Wireless Information Technology and Systems (pp. 1-4). IEEE.

[17] Raviteja, G.V., 2018. An Insight into Impact of Partial Ground and Dual Trapezoidal Slots on Bandwidth and Gain considerations for a Microstrip Antenna Array for WiMax Application. Advanced Electromagnetics, 7(5), pp.111-117. 